\title{
Germinação de megásporos e desenvolvimento esporofítico inicial da filicínea Regnellidium diphylum (Marsileaceae) sob deficiências minerais ${ }^{1}$
}

\author{
DENISE ANGELA WUNDER ${ }^{2}$, ANNETTE DROSTE ${ }^{3}$ e PAULO GÜNTER WINDISCH ${ }^{4,5}$
}

(recebido: 12 de fevereiro de 2009; aceito: 13 de maio de 2010)

\begin{abstract}
Megaspore germination and initial sporophytic development of the fern Regnellidium diphyllum (Marsileaceae) under mineral deficiencies). Regnellidium diphyllum Lindm. is a heterosporous fern in the Marsileaceae family with restricted distribution to Southern Brazil and some adjoining localities in Uruguay and Argentina. The species has been listed as vulnerable in the endangered species list of the State of Rio Grande do Sul. In the present study we verified the effect of absence of mineral nutrients on megaspore germination and initial development of $R$. diphyllum sporophytes in vitro. The effect of Meyer's nutrient solutions modified by the absence of $\mathrm{NH}_{4} \mathrm{NO}_{3}, \mathrm{NaCl}, \mathrm{FeCl}_{3} \cdot 6 \mathrm{H}_{2} \mathrm{O}, \mathrm{MgSO}_{4} \cdot 7 \mathrm{H}_{2} \mathrm{O}, \mathrm{KH}_{2} \mathrm{PO}_{4}$ or $\mathrm{CaCl}_{2}$ was analyzed in the germination and sporophyte formation of $R$. diphyllum. Megaspore germination decreased in absence of $\mathrm{CaCl}_{2}(52 \%)$ when compared to the control (77.3\%). A low percentage of gametophytes gave rise to sporophytes in the absence of $\mathrm{MgSO}_{4} \cdot 7 \mathrm{H}_{2} \mathrm{O}$ $(38.6 \%)$ and $\mathrm{KH}_{2} \mathrm{PO}_{4}(33.8 \%)$. Without $\mathrm{CaCl}_{2}$ in the solution, no sporophytes were formed. The length of the primary root and of the primary and secondary leaves was significantly reduced without $\mathrm{MgSO}_{4} \cdot 7 \mathrm{H}_{2} \mathrm{O}$ and $\mathrm{KH}_{2} \mathrm{PO}_{4}$. Without $\mathrm{KH}_{2} \mathrm{PO}_{4}$ in the solution, no secondary leaf was formed after five weeks of culture. The other treatments did not inhibit the megaspore germination process and the initial development of sporophytes, showing that the species may establish itself even under low levels of these nutrients.
\end{abstract}

Key words - aquatic macrophyte, ecophysiology, mineral nutrition, pteridophytes, reproduction

RESUMO - (Germinação de megásporos e desenvolvimento esporofítico inicial da filicínea Regnellidium diphylum (Marsileaceae) sob deficiências minerais). Regnellidium diphyllum Lindm. é uma filicínea heterosporada da família Marsileaceae, de distribuição restrita ao sul do Brasil e algumas localidades do Uruguai e da Argentina. A espécie se encontra na lista de espécies ameaçadas de extinção do Rio Grande do Sul, como vulnerável. No presente estudo, verificou-se o efeito da ausência de nutrientes minerais na germinação de megásporos e no desenvolvimento inicial de esporófitos de $R$. diphyllum in vitro. $\mathrm{O}$ efeito de soluções nutritivas de Meyer modificadas pela ausência de $\mathrm{NH}_{4} \mathrm{NO}_{3}, \mathrm{NaCl}, \mathrm{FeCl} \cdot 6 \mathrm{H}_{2} \mathrm{O}, \mathrm{MgSO}_{4} \cdot 7 \mathrm{H}_{2} \mathrm{O}, \mathrm{KH}_{2} \mathrm{PO}_{4}$ ou $\mathrm{CaCl}_{2}$ foi analisado na germinação e na formação de esporófitos de $R$. diphyllum. A germinação dos megásporos foi reduzida na ausência de $\mathrm{CaCl}_{2}(52 \%)$, quando comparada ao controle (77,3\%). Houve baixa porcentagem de gametófitos com formação de esporófitos na ausência de $\mathrm{MgSO}_{4} \cdot 7 \mathrm{H}_{2} \mathrm{O}(38,6 \%)$ e de $\mathrm{KH}_{2} \mathrm{PO}_{4}(33,8 \%)$, sendo que na ausência de $\mathrm{CaCl}_{2}$ não houve formação de esporófitos. $\mathrm{O}$ comprimento da raiz primária e das folhas primária e secundária foi reduzido significativamente na ausência de $\mathrm{MgSO}_{4} \cdot 7 \mathrm{H}_{2} \mathrm{O}$ e de $\mathrm{KH}_{2} \mathrm{PO}_{4}$, destacando-se que na ausência de $\mathrm{KH}_{2} \mathrm{PO}_{4}$ não ocorreu desenvolvimento da folha secundária após cinco semanas em cultura. Os demais tratamentos não comprometeram o processo de germinação dos megásporos e o desenvolvimento inicial dos esporófitos, demonstrando que a espécie pode se estabelecer mesmo sob baixas concentrações desses nutrientes.

Palavras-chave - ecofisiologia, macrófita aquática, nutrição mineral, pteridófitas, reprodução

1. Parte da dissertação de mestrado da primeira autora, Programa de Pós-Graduação em Biologia da Universidade do Vale do Rio dos Sinos, São Leopoldo, RS, Brasil.

2. Prefeitura Municipal de Augusto Pestana, Secretaria Municipal da Agricultura e Meio Ambiente, Rua da República 96, 98740-000 Augusto Pestana, RS, Brasil.

3. Universidade do Vale do Rio dos Sinos, Avenida Unisinos 950, 93022-000 São Leopoldo, RS, Brasil; Endereço atual: Universidade Feevale, RS239 2755, 93352-000 Novo Hamburgo, RS, Brasil.

4. Universidade Federal do Rio Grande do Sul, Programa de Pós-Graduação em Botânica, Avenida Bento Gonçalves 9500, 91501-970 Porto Alegre, RS, Brasil.

5. Autor para correspondência: pteridos@gmail.com

\section{Introdução}

A maioria dos estudos realizados com espécies de macrófitas aquáticas, entre elas algumas pteridófitas, é voltada para locais com altas concentrações de nutrientes. Experimentos realizados com as angiospermas Eichhornia crassipes (Mart.) Solms, Pistia stratiotes L. e Hydrocotyle umbellata L. demonstraram a eficiência dessas espécies na absorção de nutrientes em meio de cultura (Reddy \& Debusk 1984). Da mesma forma, Spirodela polyrhiza (L.) Schleid., Lemna minor L. e as pteridófitas Salvinia 
rotundifolia Willd. e Azolla caroliniana Willd. podem ser usadas em tratamentos de ecossistemas aquáticos eutrofizados, por absorverem altas doses de nutrientes (Reddy \& Debusk 1985, Adalberto et al. 2004).

Por outro lado, o efeito da deficiência nutricional em plantas é pouco estudado e, especificamente, para pteridófitas, as informações disponíveis são escassas. Bloom \& Voth (1956) avaliaram o crescimento de propágulos de Regnellidium diphyllum Lindm. obtidos de plantas cultivadas em tanques com solo úmido, sob diferentes combinações de cátions e ânions. Propágulos submetidos a altas concentrações de cálcio e baixas concentrações de potássio e magnésio mostraram crescimento máximo. Por outro lado, quando os propágulos foram submetidos à variação nos teores de ânions, maior crescimento foi observado na presença de baixos níveis de fósforo combinados com concentrações elevadas de nitrogênio. Vianna (1974) investigou a germinação de megásporos e o desenvolvimento de esporófitos de Regnellidium diphyllum em diferentes concentrações e composições de meio de cultura, testando a capacidade de germinação em água corrente, água destilada e soluções nutritivas de Meyer (Meyer et al. 1955) e Knop (Knop 1865).

Regnellidium diphyllum é uma filicínea heterosporada pertencente à família Marsileaceae, tradicionalmente considerada na ordem Marsileales. Atualmente é classificada como pertencente à classe Polypodiopsida, ordem Salviniales (Smith et al. 2006). Seu habitat é semi-aquático, podendo ocorrer como terrícola, em áreas periféricas; ou submersa, em áreas alagadas. Uma interessante característica reprodutiva dessa espécie é a capacidade de os megásporos darem origem a esporófitos na ausência de micrósporos, pelo processo de apogamia (Mahlberg \& Baldwin 1975). A espécie apresenta distribuição restrita ao sul do Brasil, sendo encontrada com maior freqüência no Rio Grande do Sul (Schultz 1949), mas também há registros de ocorrência em Santa Catarina (Sehnem 1979) e em algumas localidades da Argentina e do Uruguai (Sota 1977, Alonso-Paz \& Bassagoda 2002). Atualmente, encontra-se na lista de espécies ameaçadas de extinção do Rio Grande do Sul (Rio Grande do Sul 2008), como vulnerável quanto ao seu estado de conservação.

Diante do estado de conservação de Regnellidium diphyllum e da carência de estudos sobre a influência de nutrientes minerais em seu estabelecimento no habitat natural, o presente trabalho teve por objetivo verificar o efeito da ausência de nutrientes minerais sobre a germinação de megásporos e o desenvolvimento inicial de esporófitos dessa espécie, em experimentos in vitro.

\section{Material e métodos}

Os megásporos de Regnellidium diphyllum provieram de esporocarpos coletados no Município de Triunfo (2948' S, $51^{\circ} 41^{\prime} \mathrm{W}$ ), Rio Grande do Sul, Brasil, que se encontravam acondicionados no banco de germoplasma da Universidade do Vale do Rio dos Sinos, em temperatura ambiente $\left(25^{\circ} \mathrm{C}\right)$. Um espécimen testemunho (Kieling-Rubio 280) está depositado no Herbário do Instituto Anchietano de Pesquisas, São Leopoldo, RS, sigla PACA, registro 101678. Quinze esporocarpos foram esterilizados de acordo com a metodologia descrita por Wunder et al. (2009). Os esporocarpos foram rompidos e os megásporos foram isolados dos micrósporos, com o auxílio de um estereomicroscópio. Fez-se a homogeneização da amostra por meio da mistura dos megásporos dos diferentes esporocarpos. Considerando a ocorrência normal de apogamia nos megagametófitos de Regnellidium diphyllum (Mahlberg \& Baldwin 1975) apenas os megásporos foram submetidos à germinação, visando a uniformizar o processo de formação dos esporófitos, excluindo assim a possibilidade de esporófitos serem formados pelo processo sexual.

Para o cultivo dos esporocarpos, utilizou-se a solução nutritiva de Meyer (Meyer et al. 1955), com a seguinte composição: $1,0 \mathrm{~g} \mathrm{~L}^{-1} \mathrm{KH}_{2} \mathrm{PO}_{4}, 1,0 \mathrm{~g} \mathrm{~L}^{-1} \mathrm{NH}_{4} \mathrm{NO}_{3}, 0,3 \mathrm{~g} \mathrm{~L}^{-1}$ $\mathrm{MgSO}_{4} \cdot 7 \mathrm{H}_{2} \mathrm{O}, 0,06 \mathrm{~g} \mathrm{~L}^{-1} \mathrm{CaCl}_{2}, 0,1 \mathrm{~g} \mathrm{~L}^{-1} \mathrm{NaCl}$ e $0,01 \mathrm{~g} \mathrm{~L}^{-1}$ $\mathrm{FeCl}_{3} \cdot 6 \mathrm{H}_{2} \mathrm{O}$. A partir dessa solução nutritiva, foram estabelecidos sete tratamentos: $\mathrm{M}-\mathrm{A}=$ ausência de $\mathrm{NH}_{4} \mathrm{NO}_{3}$; $\mathrm{M}-\mathrm{B}=$ ausência de $\mathrm{NaCl}$; M-C $=$ ausência de $\mathrm{FeCl}_{3} \cdot 6 \mathrm{H}_{2} \mathrm{O}$; $\mathrm{M}-\mathrm{D}=$ ausência de $\mathrm{MgSO}_{4} \cdot 7 \mathrm{H}_{2} \mathrm{O} ; \quad \mathrm{M}-\mathrm{E}=$ ausência de $\mathrm{KH}_{2} \mathrm{PO}_{4} ; \mathrm{M}-\mathrm{F}=$ ausência de $\mathrm{CaCl}_{2}$ e $\mathrm{M}$ solução nutritiva de Meyer completa (controle). O pH foi ajustado para 6,2. Foram colocados vinte e cinco megásporos em cada frasco de vidro (3,5 cm de diâmetro, capacidade de $80 \mathrm{~mL}$ ) contendo $25 \mathrm{~mL}$ de solução, totalizando seis repetições (seis frascos) por tratamento. Os frascos foram mantidos em câmara de germinação do tipo BOD com fotoperíodo de 16 horas de luz, sob irradiância nominal de $110 \mu \mathrm{mol} \mathrm{m}^{-2} \mathrm{~s}^{-1} \mathrm{a} 24^{\circ} \mathrm{C}$.

Para verificar o desenvolvimento dos esporófitos de Regnellidium diphyllum, dois indivíduos de cada repetição por tratamento foram fixados em álcool $70 \%$ ao final da primeira e da quinta semana de cultura. Foi medido o comprimento da raiz primária, da folha primária e da folha secundária. Também foi monitorada a manifestação de sintomas visíveis de deficiência nutricional nas amostras dos diferentes tratamentos, por meio de imagens digitais. Após cinco semanas, avaliaram-se o total de megásporos germinados e os megagametófitos com formação de esporófito em cada tratamento, sendo feita a contagem de folhas de dois indivíduos por repetição, totalizando doze indivíduos por tratamento. Consideraram-se germinados os megásporos que emitiram uma estrutura globular clorofilada e envolta por uma coroa de rizóides, ou aqueles que já apresentavam raiz e folhas, conforme Mahlberg \& Baldwin (1975).

Verificaram-se a normalidade e a homogeneidade dos dados coletados após uma e cinco semanas, respectivamente, pelos testes de Kolmogorov-Smirnov (Santana \& Ranal 2004) 
e de Levene. Os dados foram analisados pelo teste de KruskalWallis, sendo que a diferença entre as médias foi verificada através do teste de Dunn a 5\% de probabilidade (Zar 1999). Utilizou-se o programa estatístico SYSTAT versão 10.2.

\section{Resultados e discussão}

O efeito da ausência dos diferentes nutrientes minerais sobre a porcentagem de germinação dos megásporos de Regnellidium diphyllum foi avaliado e mostrou que apenas o tratamento na ausência de $\mathrm{CaCl}_{2}$ (M-F) diferiu do tratamento controle (M) $(P=0,01)$, observando-se a menor porcentagem de megásporos germinados (52\%) (figura 1A). Esse resultado pode estar relacionado com a ausência de cálcio, que é um macroelemento essencial na síntese de novas paredes celulares, nas divisões celulares, no funcionamento normal das membranas, na formação de raízes, caules e folhas, além de mensageiro secundário nas respostas mediadas por hormônios vegetais e pela luz (Salisbury \& Ross 1991). Geralmente, as plantas presentes em meio deficiente em cálcio sofrem necrose em regiões meristemáticas jovens (Berry 2006) e, por esse motivo, o desenvolvimento das estruturas esporofíticas pode ter sido prejudicado. Por outro lado, o processo de germinação não foi afetado pelos demais nutrientes avaliados, o que deve estar relacionado ao fato de os megásporos das diferentes espécies de Marsileaceae conterem numerosos grãos de amido e menores grânulos de proteínas e lipídios (Higinbotham 1941), substâncias de reserva que permitem a germinação mesmo na ausência de certos nutrientes ou apenas em água (Vianna 1974).

Os megásporos germinados na ausência de $\mathrm{CaCl}_{2}$ (M-F) originaram estruturas clorofiladas nos primeiros dias em cultura, as quais, no entanto, necrosaram e não formaram esporófitos. Nas soluções nutritivas destituídas de $\mathrm{MgSO}_{4} \cdot 7 \mathrm{H}_{2} \mathrm{O}$ (M-D) e $\mathrm{KH}_{2} \mathrm{PO}_{4}$ (M-E), foram obtidas porcentagens de gametófitos com esporófito estatisticamente menores do que no controle $(38,6 \%$ e 33,8\%, respectivamente). Os demais tratamentos apresentaram porcentagens de esporófitos formados similares ao tratamento controle (figura 1B).

O desenvolvimento das estruturas esporofíticas (raiz primária, folha primária e folha secundária) de Regnellidium diphyllum foi influenciado pelos diferentes tratamentos. O comprimento da raiz primária foi significativamente menor $(P<0,001)$ na ausência de $\mathrm{MgSO}_{4} \cdot 7 \mathrm{H}_{2} \mathrm{O}(\mathrm{M}-\mathrm{D})$ e de $\mathrm{KH}_{2} \mathrm{PO}_{4}$ (M-E). Especificamente, na ausência de $\mathrm{MgSO}_{4} \cdot 7 \mathrm{H}_{2} \mathrm{O}$ (M-D), foram observados megásporos germinados envoltos por uma coroa de rizóides, que, no entanto, não apresentavam
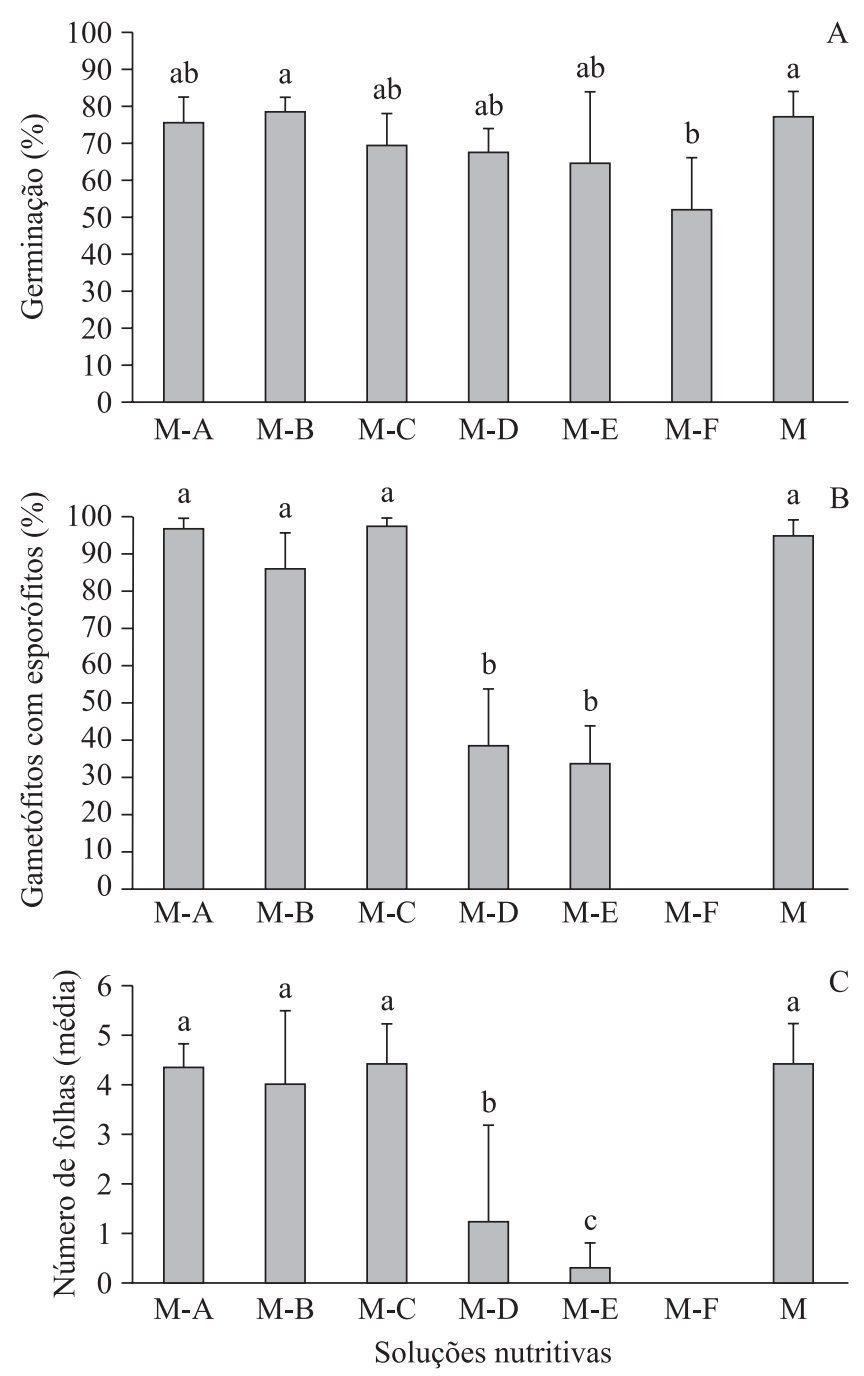

Figura 1. A. Germinação de megásporos (médias \pm desvios padrões) $(H=16,812, g l=6,35 P=0,01)(n=6 \times 25)$. B. Formação de esporófitos (médias \pm desvios padrões) $(H=25,264, g l=5,30, P<0,001)(n=6 \times 25)$. C. Número de folhas em esporófitos (médias \pm desvios padrões) $(H=39,210$, $g l=5,30, P<0,001)(n=12 \times 6)$ de Regnellidium diphyllum após cinco semanas em cultura na ausência dos seguintes minerais da solução de Meyer. $\mathrm{M}-\mathrm{A}=\mathrm{NH}_{4} \mathrm{NO}_{3} ; \mathrm{M}-\mathrm{B}=\mathrm{NaCl}$; $\mathrm{M}-\mathrm{C}=\mathrm{FeCl}_{3} \cdot 6 \mathrm{H}_{2} \mathrm{O} ; \mathrm{M}-\mathrm{D}=\mathrm{MgSO}_{4} \cdot 7 \mathrm{H}_{2} \mathrm{O} ; \mathrm{M}-\mathrm{E}=\mathrm{KH}_{2} \mathrm{PO}_{4}$, $\mathrm{M}-\mathrm{F}=\mathrm{CaCl}_{2} . \mathrm{M}=$ solução de Meyer completa (controle).

Figure 1. A. Megaspore germination (means \pm standard deviations) $(H=16.812, g l=6,35 P=0.01)(n=6 \times 25)$. B. Sporophyte formation (means \pm standard deviations) $(H=25.264, g l=5,30, P<0.001)(n=6 \times 25)$. C. Leafnumber in sporophytes (means \pm standard deviations) $(H=39.210$, $g l=5,30, P<0.001)(n=12 \times 6)$ of Regnellidium diphyllum after five weeks in culture in the absence of the following mineral nutrients of Meyer's solution. $\mathrm{M}-\mathrm{A}=\mathrm{NH}_{4} \mathrm{NO}_{3}$; $\mathrm{M}-\mathrm{B}=\mathrm{NaCl} ; \quad \mathrm{M}-\mathrm{C}=\mathrm{FeCl}_{3} \cdot 6 \mathrm{H}_{2} \mathrm{O} ; \quad \mathrm{M}-\mathrm{D}=\mathrm{MgSO}_{4} \cdot 7 \mathrm{H}_{2} \mathrm{O}$; $\mathrm{M}-\mathrm{E}=\mathrm{KH}_{2} \mathrm{PO}_{4}, \quad \mathrm{M}-\mathrm{F}=\mathrm{CaCl}_{2} . \quad \mathrm{M}=$ Meyer's complete solution (control). 
estrutura esporofítica desenvolvida. Por outro lado, na ausência de $\mathrm{NH}_{4} \mathrm{NO}_{3}$ (M-A), houve crescimento acelerado da raiz primária, que atingiu 16,5 mm após cinco semanas, comprimento quatro vezes maior quando comparado à média do comprimento da estrutura no tratamento controle (4,08 mm). Na ausência de $\mathrm{NaCl}(\mathrm{M}$ B) e de $\mathrm{FeCl}_{3} \cdot 6 \mathrm{H}_{2} \mathrm{O}$ (M-C), as médias do comprimento da raiz primária foram semelhantes ao tratamento controle (tabela 1).

O comprimento da folha primária também foi significativamente afetado pela ausência de nutrientes $(P<0,001)$. Esta estrutura apresentou reduzido crescimento na ausência de $\mathrm{MgSO}_{4} \cdot 7 \mathrm{H}_{2} \mathrm{O}(\mathrm{M}-\mathrm{D})$ e $\mathrm{KH}_{2} \mathrm{PO}_{4}$ (M-E), sendo que as médias diferiram do tratamento controle. $\mathrm{Na}$ ausência de $\mathrm{NH}_{4} \mathrm{NO}_{3}$ (M-A), constatou-se que, após uma semana, o comprimento da folha primária foi estatisticamente superior àquele encontrado nos demais tratamentos, o que não se confirmou após cinco semanas (tabela 1). A presença de compostos nitrogenados na solução nutritiva pode ter inibido inicialmente o crescimento foliar, pois o $\mathrm{NH}_{4}^{+}$ é absorvido pelas raízes por processo passivo quando a concentração externa é alta, e seu acúmulo pode prejudicar a planta (Britto et al. 2001).

A ausência de nutrientes minerais ainda influenciou negativamente o comprimento da folha secundária $(P<0,001)$. Durante a primeira semana, não houve formação destas folhas na ausência de $\mathrm{MgSO}_{4} \cdot 7 \mathrm{H}_{2} \mathrm{O}$ (M-D) e de $\mathrm{KH}_{2} \mathrm{PO}_{4}$ (M-E). Após cinco semanas, na ausência de $\mathrm{MgSO}_{4} \cdot 7 \mathrm{H}_{2} \mathrm{O}$ (M-D), as folhas secundárias apresentaram uma redução substancial de seu comprimento (média de 1,91 $\mathrm{mm}$ ) quando comparadas às folhas secundárias no tratamento controle (média de $10,08 \mathrm{~mm}$ ). $\mathrm{Na}$ ausência de $\mathrm{KH}_{2} \mathrm{PO}_{4}$ (M-E), mesmo após cinco semanas, não ocorreu formação de folhas secundárias. Não houve diferença estatisticamente significativa do comprimento da folha secundária entre os demais tratamentos, incluindo o controle (tabela 1).

Após cinco semanas, verificaram-se indícios de clorose nas folhas mais velhas de esporófitos cultivados em todos os tratamentos, excetuando o controle. Os nutrientes que causaram os sintomas mais evidentes de clorose com evolução para necrose, foram $\mathrm{o}$ $\mathrm{MgSO}_{4} \cdot 7 \mathrm{H}_{2} \mathrm{O}$ (M-D) e o $\mathrm{KH}_{2} \mathrm{PO}_{4}$ (M-E), evidenciando a baixa tolerância da espécie frente à retirada destes da solução nutritiva. Também o número de folhas por indivíduo foi significativamente menor $(P<0,001)$ nesses tratamentos (figura 1C).

A sensibilidade apresentada pela espécie à ausência de nutrientes no presente estudo não necessariamente está relacionada apenas à supressão individual de sais minerais do meio de cultura em cada tratamento, mas também pode estar ligada às possíveis interações entre os diversos nutrientes, uma vez que a quantidade de um

Tabela 1. Crescimento de Regnellidium diphyllum (média \pm desvio padrão do comprimento da raiz primária e das folhas primária e secundária) após uma e cinco semanas em cultura na ausência dos seguintes nutrientes minerais da solução de Meyer: $\mathrm{M}=$ solução de Meyer completa (controle); $\mathrm{M}-\mathrm{A}=\mathrm{NH}_{4} \mathrm{NO}_{3} ; \mathrm{M}-\mathrm{B}=\mathrm{NaCl} ; \mathrm{M}-\mathrm{C}=\mathrm{FeCl}_{3} \cdot 6 \mathrm{H}_{2} \mathrm{O} ; \mathrm{M}-\mathrm{D}=\mathrm{MgSO}_{4} \cdot 7 \mathrm{H}_{2} \mathrm{O}$; $\mathrm{M}-\mathrm{E}=\mathrm{KH}_{2} \mathrm{PO}_{4}$. $\mathrm{O}$ valor de $H$ corresponde ao teste de Kruskal-Wallis. Diferentes letras, na vertical, denotam diferenças significativas entre tratamentos pelo Teste de Dunn $(P<0,05 ; n=12)$.

Table 1. Regnellidium diphyllum growth (length mean \pm standard deviation of primary root, primary leaf and first secondary leaf) after one and five weeks in culture in the absence of the following mineral nutrients of Meyer's solution: $\mathrm{M}=$ complete Meyer's solution (control); $\mathrm{M}-\mathrm{A}=\mathrm{NH}_{4} \mathrm{NO}_{3} ; \mathrm{M}-\mathrm{B}=\mathrm{NaCl} ; \mathrm{M}-\mathrm{C}=\mathrm{FeCl}_{3} \cdot 6 \mathrm{H}_{2} \mathrm{O} ; \mathrm{M}-\mathrm{D}=\mathrm{MgSO}_{4} \cdot 7 \mathrm{H}_{2} \mathrm{O} ; \mathrm{M}-\mathrm{E}=\mathrm{KH}_{2} \mathrm{PO}_{4}$. $H$ value corresponds to Kruskal-Wallis test. Different letters in the vertical denote significant differences among treatments according to Dunn test $(P<0.05 ; n=12)$.

\begin{tabular}{|c|c|c|c|c|c|c|}
\hline \multirow{2}{*}{ Tratamento } & \multicolumn{2}{|c|}{ Raiz primária (mm) } & \multicolumn{2}{|c|}{ Folha primária $(\mathrm{mm})$} & \multicolumn{2}{|c|}{ Folha secundária (mm) } \\
\hline & 1 semana & 5 semanas & 1 semana & 5 semanas & 1 semana & 5 semanas \\
\hline M & $2,83 \pm 1,48 \mathrm{~b}$ & $4,08 \pm 1,73 b$ & $5,41 \pm 2,23 b$ & $8,00 \pm 2,17 \mathrm{a}$ & $0,76 \pm 0,59 \mathrm{ab}$ & $10,08 \pm 2,87 \mathrm{a}$ \\
\hline M-A & $10,54 \pm 2,36 \mathrm{a}$ & $16,50 \pm 6,38 \mathrm{a}$ & $8,08 \pm 1,01 \mathrm{a}$ & $7,66 \pm 1,61 \mathrm{a}$ & $1,20 \pm 0,31 \mathrm{a}$ & $9,25 \pm 1,96 \mathrm{a}$ \\
\hline M-B & $3,04 \pm 2,17 \mathrm{~b}$ & $3,54 \pm 2,64 b$ & $5,83 \pm 1,64 b$ & $7,25 \pm 2,63 \mathrm{a}$ & $0,50 \pm 0,47 \mathrm{~b}$ & $6,25 \pm 3,19 \mathrm{a}$ \\
\hline $\mathrm{M}-\mathrm{C}$ & $2,66 \pm 1,09 b$ & $3,08 \pm 1,31 \mathrm{~b}$ & $5,62 \pm 1,43 b$ & $6,50 \pm 2,11 a$ & $0,87 \pm 0,31 \mathrm{ab}$ & $7,00 \pm 4,63 \mathrm{a}$ \\
\hline M-D & $1,16 \pm 0,88 \mathrm{c}$ & $1,04 \pm 1,71 \mathrm{c}$ & $1,62 \pm 0,64 \mathrm{c}$ & $2,12 \pm 2,63 b$ & $0,00 \mathrm{c}$ & $1,91 \pm 3,52 b$ \\
\hline M-E & $0,16 \pm 0,24 \mathrm{~d}$ & $0,20 \pm 0,39 \mathrm{c}$ & $0,35 \pm 0,52 \mathrm{~d}$ & $0,41 \pm 0,79 b$ & $0,00 \mathrm{c}$ & $0,00 \mathrm{c}$ \\
\hline & $\begin{array}{c}H=54,585 \\
g l=5,30 \\
P<0,001\end{array}$ & $\begin{array}{c}H=54,184 \\
g l=5,30 \\
P<0,001\end{array}$ & $\begin{array}{c}H=56,017 \\
g l=5,30 \\
P<0,001\end{array}$ & $\begin{array}{c}H=43,494 \\
g l=5,30 \\
P<0,001\end{array}$ & $\begin{array}{c}H=12,506 \\
g l=5,30 \\
P<0,001\end{array}$ & $\begin{array}{c}H=23,236 \\
g l=5,30 \\
P<0,001\end{array}$ \\
\hline
\end{tabular}


nutriente absorvida pela planta pode ser influenciada pela presença de outros (Coelho \& Verlengia 1973). $\mathrm{Na}$ natureza, a resposta fisiológica da planta é ainda mais complexa, devido à disponibilidade simultânea de diferentes nutrientes em ambientes aquáticos e terrestres ser bastante variável ao longo do tempo (Rubio et al. 2003).

Considerando que as alterações das condições nutricionais do substrato natural de Regnellidium diphyllum pela destruição ou alteração das áreas úmidas por atividades agrícolas e pecuárias são um importante fator contribuinte para a vulnerabilidade da espécie, os dados obtidos são relevantes para planos de manejo visando à conservação da espécie no habitat natural.

Agradecimentos - Os autores agradecem à Universidade do Vale do Rio dos Sinos, pelo uso dos laboratórios e pelo auxílio financeiro concedido à primeira autora; à Universidade Feevale, à Universidade Federal do Rio Grande do Sul e ao Conselho Nacional de Desenvolvimento Científico e Tecnológico (CNPq) pela subvenção.

\section{Referências bibliográficas}

ADALBERTO, P.R., MASSABNI, A.C., GOULART, A.J., MONTI, R. \& LACAVA, P.M. 2004. Efeito do fósforo na captação de minerais e pigmentação de Azolla caroliniana Willd. (Azollaceae). Revista Brasileira de Botânica 27:581-585.

ALONSO-PAZ, E. \& BASSAGODA, M.J. 2002. Revisión de las Marsileaceae del Uruguay y primera cita de Pilularia americana A. Braun. Comunicaciones Botanicas - Museo Nacional de Historia Natural y Antropologia $6: 1-8$.

BERRY, W. 2006. Symptoms of deficiency in essential minerals. Topic 5.1. In Plant Physiology Online (L.Taiz \& E. Zeiger, eds.). http://4e.plantphys.net/ (acesso em 04/01/2009).

BLOOM, W.W. \& VOTH, P.D. 1956. Responses of Regnellidium diphyllum to nutrient supply and photoperiod. Botanical Gazette 117:173-193.

BRITTO, D.T., SIDDIQI, M.Y., GLASS, A.D.M. \& KRONZUCKER, H.J. 2001. Futile transmembrane $\mathrm{NH}_{4}^{+}$cycling: a cellular hypothesis to explain ammonium toxicity in plants. Proceeding of the National Academy of Sciences 98:4255-4258.

COELHO, F.S. \& VERLENGIA, F. 1973. Fertilidade do solo. Instituto Campineiro de Ensino Agrícola, São Paulo.

HIGINBOTHAM, N. 1941. Development of the gametophytes and embryo of Regnellidium diphyllum. American Journal of Botany 28:282-300.
KNOP, W. 1865. Quantitative Untersuchungen über den Ernährungs-Prozess der Pflanzen. Landwirtschaftliche Versuchsstation Poland 7:93-107.

MAHLBERG, P.G. \& BALDWIN, M. 1975. Experimental studies on megaspore viability, partenogenesis and sporophyte formation in Marsilea, Pilularia and Regnellidium. Botanical Gazette 136:269-273.

MEYER, B.S., ANDERSON, D.B. \& SWANSON, C.A. 1955. Laboratory plant physiology. Van Nostrand, New York.

REDDY, K.R. \& DEBUSK, W.F. 1984. Growth characteristics of aquatic macrophytes cultured in nutrient-enriched water: I. Water hyacinth, water letuce, and pennywort. Economic Botany 38:229-239.

REDDY, K.R. \& DEBUSK, W.F. 1985. Growth characteristics of aquatic macrophytes cultured in nutrient-enriched water: II. Azolla, Dukweed, and Salvinia. Economic Botany 39:200-208.

RIO GRANDE DO SUL. Secretaria do Meio Ambiente. 2008. Espécies da flora ameaçadas de extinção do Rio Grande do Sul. Secretaria do Meio Ambiente, Porto Alegre.

RUBIO, G., ZHU, J. \& LYNCH, J.P. 2003. A critical test of the two prevailing theories of plant response to nutrient availability. American Journal of Botany 90:143-152.

SALISBURY, F.B. \& ROSS, C.W. 1991. Plant Physiology. $4^{\text {th }}$ ed. Wadsworth, Belmont.

SANTANA, D.G. \& RANAL, M.A. 2004. Análise da germinação: um enfoque estatístico. Universidade de Brasília, Brasília.

SCHULTZ, A.R. 1949. Contribuições ao conhecimento de Regnellidium diphyllum Lindm. Lilloa 27:139-144.

SEHNEM, A. 1979. Marsileáceas. In Flora Ilustrada Catarinense (R. Reitz, ed). Herbário Barbosa Rodrigues, Itajaí, p.1-12.

SMITH, A.R., PRYER, K.M., SCHUETTPELZ, E., KORALL, P., SCHNEIDER, H. \& WOLF, P.G. 2006. A classification for extant ferns. Taxon 55:705-731.

SOTA, E.R. DE LA. 1977. Pteridophyta. In Flora de la Provincia de Jujuy, Republica Argentina (A. Cabrera, ed.). Instituto Nacional de Tecnologia e Agropecuária (INTA), Buenos Aires, p.1-18.

VIANNA, E.C. 1974. Nota sobre o desenvolvimento de Regnellidium diphyllum Lindm. (Marsileaceae). Iheringia - Série Botânica 19:25-30.

WUNDER, D.A., DROSTE, A. \& WINDISCH, P.G. 2009. Megaspore germination and initial development of Regnellidium diphyllum Lindman (Pteridophyta, Marsileaceae) sporophytes in the presence of cadmium. Revista Brasileira de Botânica 32:177-181.

ZAR, J.H. 1999. Biostatistical analyses. $4^{\text {th }}$ ed., Prentice-Hall, Upper Saddle River. 